\title{
Successful fertility treatment with gonadotrophin therapy for male hypogonadotrophic hypogonadism
}

\author{
J Rajkanna, S Tariq and S O Oyibo \\ Department of Endocrinology, Peterborough City Hospital, Bretton Gate, Peterborough PE3 9GZ, UK
}

Correspondence

should be addressed

to S O Oyibo

Email

samoyibo@yahoo.co.uk

\section{Summary}

Gonadotrophin therapy with human chorionic gonadotrophin and recombinant FSH is indicated for use in men with reduced spermatogenesis due to hypogonadotrophic hypogonadism $(\mathrm{HH})$. Patients require regular monitoring for side effects and desired response to treatment. We present a man with $\mathrm{HH}$, azoospermia and a history of previous anabolic steroid usage who had undergone gonadotrophin therapy, had subsequently achieved conception and has now fathered a child.

\section{Learning points:}

- In total, $15 \%$ of couples do not achieve pregnancy within 1 year and seek medical treatment for infertility: male factors contribute to $50 \%$ of these.

- The evaluation of male infertility should include a full history and examination, an endocrine profile and a quality-controlled semen analysis.

- HH with defective spermatogenesis is an important cause of male infertility in a small percentage of cases.

- Gonadotrophin therapy requires regular monitoring for side effects and desired response to treatment.

- Any sustained rise in prostate specific antigen levels should prompt urological assessment for possible prostate biopsy.

- A multidisciplinary approach is required for gonadotrophin therapy, especially if assisted fertilisation techniques are required once, spermatogenesis is achieved.

\section{Background}

Infertility affects $15 \%$ of couples, and a male factor is a contributory cause in $50 \%$ of cases (1). Adequate history taking, examination of both partners and hormone testing and semen analysis are required to ascertain the cause and possible treatment strategies (1).

Of the several factors responsible for male infertility, hypogonadotrophic hypogonadism $(\mathrm{HH})$ is a treatable cause in a small number of individuals. $\mathrm{HH}$ can be congenital (e.g. Kallmann syndrome) or acquired due to a pituitary lesion, hyperprolactinaemia or previous anabolic steroids abuse. Patients have reduced testosterone levels and spermatogenesis (oligospermia or azoospermia) secondary to reduced pituitary gonadotrophins, i.e. luteinizing hormone (LH) and follicle-stimulating hormone (FSH). LH is required to stimulate the Leydig cells in the testis to produce testosterone which acts on the Sertoli cells to stimulate sperm production. FSH directly stimulates the Sertoli cells to support sperm production. Therefore, patients with $\mathrm{HH}$ require gonadotrophin therapy to stimulate spermatogenesis, after detailed imaging of their pituitary gland. 


\section{Case presentation}

A 25-year-old gentleman was presented to the endocrine clinic in November 2013 with a history of tiredness, reduced libido and inability to father a child for more than a year. He had a child with a previous partner, and his current female partner had already been fully evaluated by the fertility specialist.

In 2007, he took anabolic steroids during bodybuilding training, but stated that he had symptoms of tiredness and reduced libido before then. In 2011, he stopped taking the steroids because he wanted to father a child with his partner. After a year of no success, he and his partner decided to seek fertility treatment.

On examination, he was well with normal secondary sexual features and normal looking external genitalia (mean testicular volume of $20 \mathrm{ml}$ ). There were no previous investigation results to suggest hypogonadism predated anabolic steroid abuse.

After baseline investigations, he started gonadotrophin therapy. He had 3-4 monthly investigations to monitor for side effects, hormonal response to treatment and for evidence of spermatogenesis.

Once adequate spermatogenesis was confirmed by serial semen analysis and because there was delayed success with non-assisted conception, his stored frozen semen samples were used for assisted conception treatment.

\section{Investigation}

Results of baseline and follow-up investigations are shown in Table 1. Baseline tests revealed $\mathrm{HH}$ as well as azoospermia on two separate occasions. Magnetic resonance imaging demonstrated a normal pituitary gland. Appreciable spermatogenesis was observed at 9 and 12 months after starting gonadotrophin therapy.

\section{Treatment}

This consisted of s.c. human chorionic gonadotrophin (HCG) 1500 IU three times a week until serum testosterone levels normalised. A negative semen analysis (at month 5) prompted the addition of s.c. rFSH $150 \mathrm{IU}$ three times a week while the dose of HCG was reduced to 1500 IU twice a week. At month 6, the HCG was stopped temporarily because of raised prostate specific antigen (PSA) levels, which returned to normal within 6 weeks. The HCG was reintroduced at a lower dose of 1500 IU once a week. Combined therapy was continued until after appreciable levels of spermatogenesis were achieved for
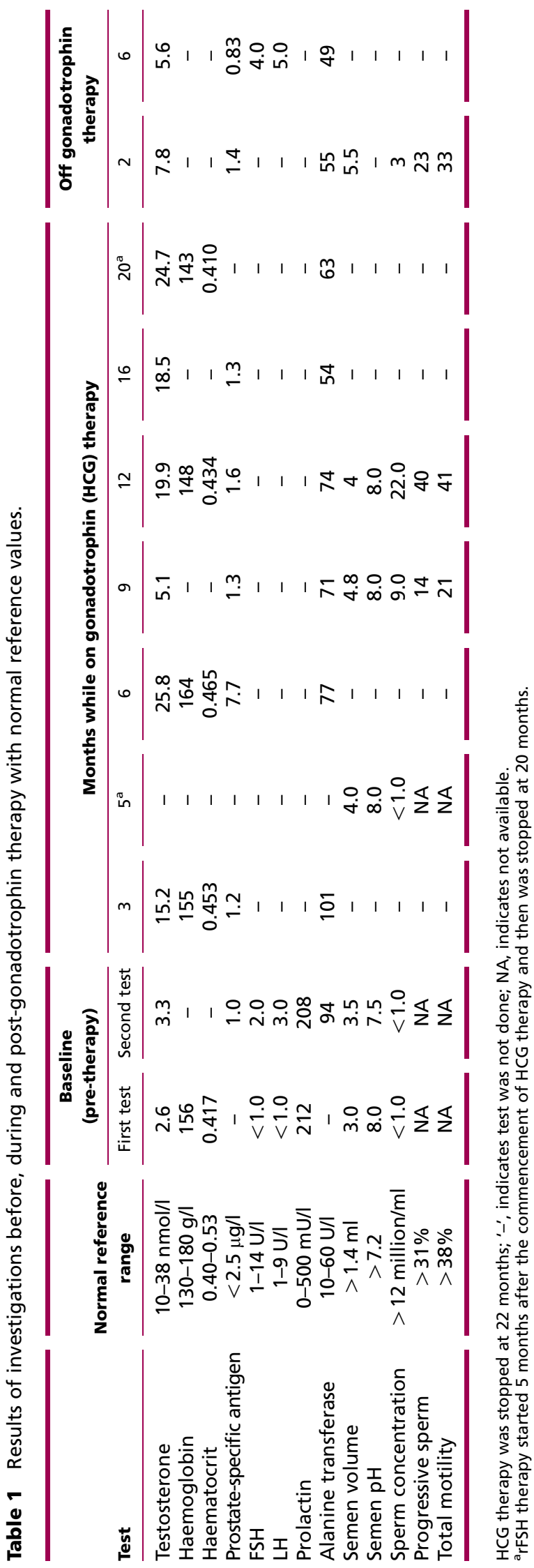

http://www.edmcasereports.com 
non-assisted conception and/or for semen freezing and assisted conception treatment. The rFSH was stopped when non-assisted conception failed and enough frozen semen samples were stored. The HCG was stopped soon after a viable pregnancy was confirmed.

\section{Outcome and follow-up}

After several months of unsuccessful non-assisted conception, the patient subsequently achieved conception through assisted conception treatment on the second attempt using his stored frozen semen sample. He now has a child. Soon after stopping gonadotrophin therapy, his testosterone levels declined to 7.8 and $5.6 \mathrm{nmol} / 1$ at 2 and 6 months respectively. He is now on testosterone replacement therapy.

\section{Discussion}

Gonadotrophin therapy (HCG/rFSH) is well tolerated and effective in inducing testis growth, spermatogenesis and fertility in gonadotrophin-deficient men (2). The availability of assisted reproduction (conception) techniques has improved the success rate even in the presence of subnormal sperm counts. However, high testicular volume at baseline, no history of cryptorchidism, a history of sexual maturation and no previous testosterone replacement therapy are positive predictors of success with gonadotrophin therapy (3). Pregnancy rates of $50-80 \%$ can be anticipated during therapy (3).

After baseline investigations, gonadotrophin therapy starts with s.c. injections of HCG three times a week until testosterone levels are high enough to induce spermatogenesis, followed by the addition or substitution of s.c. injections of rFSH to support and maintain spermatogenesis. Therapy can take 9-18 months, and spermatogenesis can be observed in more than $80 \%$ of men using this regimen (3) (4) (5). Gonadotrophin-releasing hormone infusion pump therapy is an alternative form of treatment if injection therapy has failed, but its use is limited by expense and inconvenience (6). Despite the availability of gonadotrophin therapy for treating infertility in men with $\mathrm{HH}$, there are still no standard treatment protocols available for clinical use.

Men on gonadotrophin therapy must be committed to self-injecting and being monitored for side effects of therapy and evidence of spermatogenesis every 3-4 months. Our patient's testosterone level normalised 3 months after starting HCG therapy, and an appreciable spermatogenesis was observed at 9 and 12 months (combination therapy started at month 5). During therapy, his PSA levels went above the normal range, but normalised after stopping and restarting HCG at a lower dose (1500 IU a week). PSA production from prostate epithelium is dependent on the availability of androgens; so, the levels are lower in male patients with hypogonadism compared to controls. Although PSA level usually rise within a few months of starting gonadotrophin or testosterone therapy, any sustained rise should prompt urological assessment (7) (8).

Our patient had stored frozen semen samples and stopped rFSH treatment before the first attempt at assisted conception treatment. He continued low-dose HCG treatment until stopping it soon after the second assisted conception treatment was successful. It may be prudent to stop therapy after the first trimester when the chance of miscarriage is less likely. However, spermatogenesis can occasionally be maintained with low-dose HCG alone so as to enable a subsequent pregnancy in the case of a miscarriage, but this effect may not be sustained without the use of rFSH in combination (9). Spermatogenesis can occasionally persist after stopping gonadotrophin therapy. If patients are not contemplating subsequent pregnancies, then they can commence testosterone replacement therapy. The use of stored frozen semen samples and the re-initiation of gonadotrophin therapy are important options to consider if future pregnancy is contemplated (3). Further guidance is required concerning what to do after pregnancy has been achieved, as our patient has expressed his desire to continue low-dose HCG therapy to maintain spermatogenesis.

Through negative feedback on the hypothalamicpituitary axis and gonadotrophin-releasing hormone release, prolonged anabolic steroid abuse inhibits FSH and $\mathrm{LH}$ production. This results in low testosterone levels and reduced spermatogenesis (10). Literature is sparse concerning recovery of spermatogenesis after cessation of anabolic steroid abuse and infertility treatment thereof. Recovery of spermatogenesis after cessation of exogenous steroids can take 6-12 months with subsequent achievement of conception. Some may require gonadotrophin therapy (10). Our patient required gonadotrophin therapy and had a good response to this. However, his testosterone level and semen quality began to decline soon after stopping gonadotrophin therapy, necessitating the need for testosterone replacement therapy thereafter.

There will be psychosocial issues associated with infertility treatment. Gonadotrophin therapy requires commitment even with the knowledge that there is still a failure rate depending on pre-therapy conditions. 
Gonadotrophin therapy is also expensive. Therefore, patients require adequate information and multidisciplinary team support during therapy.

In conclusion, we describe a man with $\mathrm{HH}$, azoospermia and a history of previous anabolic steroid usage who had undergone gonadotrophin therapy, had subsequently achieved conception and has now fathered a child.

\section{Patient's perspective}

All in all I am very happy with my treatment due to its success. My ideas and knowledge in this area were listened to and incorporated into my treatment. I felt the regular blood tests were laborious, but necessary in assessing my liver and prostate function levels. When it became time to provide sperm samples for private IVF treatment, the levels were more than adequate resulting in pregnancy and the subsequent birth of my daughter.

Declaration of interest

The authors declare that there is no conflict of interest that could be perceived as prejudicing the impartiality of the research reported.

\section{Funding}

This research did not receive any specific grant from any funding agency in the public, commercial or not-for-profit sector.

\section{Patient consent}

Written informed consent was obtained from the patient for publication of the submitted article and any accompanying images. The signed copy of the consent form was provided.

\section{Author contribution statement}

S O Oyibo identified the case, while J Rajkanna and S Tariq contributed to preparing the original manuscript. S O Oyibo edited the final version and is also the named physician for the patient.

\section{References}

1 Kamel RM 2010 Management of the infertile couple: an evidence-based protocol. Reproductive Biology and Endocrinology 10 21. (doi:10.1186/ 1477-7827-8-21)

2 Liu PY, Turner L, Rushford D, Baker HW, Conway AJ \& Handelsman D 1999 Efficacy and safety of recombinant human follicle stimulating hormone (Gonal-F) with urinary human chorionic gonadotropin for induction of spermatogenesis and fertility in gonadotropin-deficient men. Human Reproduction 14 1540-1545. (doi:10.1093/humrep/14. 6.1540)

3 Han TS \& Bouloux PM 2010 What is the optimal therapy for young males with hypogonadotropic hypogonadism? Clinical Endocrinology $\mathbf{7 2}$ 731-737. (doi:10.1111/j.1365-2265.2009.03746.x)

4 Zitmann M \& Nieschlag E 2000 Hormone substitution in male hypogonadism. Molecular and Cellular Endocrinology 161 73-88. (doi:10.1016/S0303-7207(99)00227-0)

5 Haidl G 2002 Management strategies for male factor infertility. Drugs 62 1741-1753. (doi:10.2165/00003495-200262120-00004)

6 Liu L, Banks SM, Barnes KM \& Sherins RJ 1988 Two-year comparison of testicular responses to pulsatile gonadotropin-releasing hormone and exogenous gonadotropins from the inception of therapy in men with isolated hypogonadotropic hypogonadism. Journal of Clinical Endocrinology and Metabolism 67 1140-1145. (doi:10.1210/jcem-67-6-1140)

7 Ozata M, Bulur M, Beyhan Z, Sengül A, Saglam M, Turan M, Corakci A \& Ali Gundogan M 1997 Effects of gonadotropin and testosterone treatments on prostate volume and serum prostate specific antigen levels in male hypogonadism. Endocrine Journal 44 719-724. (doi:10.1507/endocrj.44.719)

8 Guay AT, Perez JB, Fitaihi WA \& Vereb M 2000 Testosterone treatment in hypogonadal men: prostate-specific antigen level and risk of prostate cancer. Endocrine Practice 6 132-138. (doi:10.4158/EP.6.2.132)

9 Depenbusch M, von Eckardstein S, Simoni M \& Nieschag E 2002 Maintenance of spermatogenesis in hypogonadotropic hypogonadal men with human chorionic gonadotropin alone. European Journal of Endocrinology/European Federation of Endocrine Societies 147 617-624. (doi:10.1530/eje.0.1470617)

10 Crosnoe LE, Grober E, Ohl D \& Kim ED 2013 Exogenous testosterone: a preventable cause of male infertility. Translational Andrology and Urology 2 106-113. (doi:10.3978/j.issn.2223-4683.2013.06.01)

Received in final form 24 December 2015 Accepted 7 January 2016 